\title{
ON $\Lambda$-STATISTICAL CONVERGENCE OF ORDER $\alpha$ IN RANDOM 2-NORMED SPACE
}

\author{
CHAWLA MEENAKSHI, M. S. SAROA, AND V. KUMAR \\ Received 24 September, 2013
}

\begin{abstract}
Let $\lambda=\left(\lambda_{n}\right)$ be a non-decreasing sequence of positive real numbers tending to $\infty$ with $\lambda_{n+1} \leq \lambda_{n}+1, \lambda_{1}=1$. In the present paper, we introduce the notion of $\Lambda$-statistical convergence of order $\alpha, \Lambda$-statistical Cauchy sequences of order $\alpha$ in random 2-normed spaces and obtain some results. We display examples which show that our method of convergence is more general in random 2-normed space.
\end{abstract}

2010 Mathematics Subject Classification: 40A05; 46A70; 46A99; 60B99

Keywords: statistical convergence, $\lambda$-statistical convergence, $t$-norm, 2-norm, random 2-normed space

\section{INTRODUCTION AND BACKGROUND}

The idea of the statistical convergence was given by Zygmund [25] in the first edition of his monograph published in Warsaw in 1935. The concept of statistical convergence was introduced by Fast [5] and Steinhaus [23] and then reintroduced by Schoenberg [20] independently. Over the years, statistical convergence has been developed in $[3,6,7,12,16,17,24]$ and turned out very useful to resolve many convergence problems arising in Analysis.

Definition 1 ([5]). A sequence $x=\left(x_{k}\right)$ of numbers is said to be statistically convergent to a number $L$ if for every $\epsilon>0$,

$$
\lim _{n \rightarrow \infty} \frac{1}{n}\left|\left\{k \leq n:\left|x_{k}-L\right|>\epsilon\right\}\right|=0,
$$

where vertical bars denotes the cardinality of enclosed set. In this case, we write $S-\lim _{k \rightarrow \infty} x_{k}=L$.

In literature, several interesting generalizations of statistical convergence have been appeared. One among these is $\lambda$-statistical convergence given by Mursaleen [14] with the help of a non-decreasing sequence $\lambda=\left(\lambda_{n}\right)$. Let $\lambda=\left(\lambda_{n}\right)$ be a nondecreasing sequence of positive real numbers tending to $\infty$ with

$$
\lambda_{n+1} \leq \lambda_{n}+1, \lambda_{1}=1 .
$$


The idea of $\lambda$-statistical convergence can be connected to the generalized de la ValléePoussin mean. It is defined by

$$
t_{n}(x)=\frac{1}{\lambda_{n}} \sum_{k \in I_{n}} x_{k}, \quad \text { where } I_{n}=\left[n-\lambda_{n}+1, n\right] \text { for } n=1,2, \ldots
$$

Definition 2 ([14]). A sequence $x=\left(x_{k}\right)$ of numbers is said to be $\lambda$-statistically convergent to a number $L$ provided that for every $\epsilon>0$,

$$
\lim _{n \rightarrow \infty} \frac{1}{\lambda_{n}}\left|\left\{k \in I_{n}:\left|x_{k}-L\right| \geq \epsilon\right\}\right|=0 .
$$

In this case, the number $L$ is called $\lambda$-statistical limit of the sequence $x=\left(x_{k}\right)$ and we write $S_{\lambda}-\lim _{k \rightarrow \infty} x_{k}=L$.

Recently, for $\alpha \in(0,1]$ Çolak and Bektaş[2] generalized Definition 2 in terms of $\lambda$-statistical convergence of order $\alpha$ and obtained some analogous results.

Definition 3 ([2]). Let $\lambda=\left(\lambda_{n}\right)$ be a non-decreasing sequence of positive real numbers as defined above and $0<\alpha \leq 1$ be given. A sequence $x=\left(x_{k}\right)$ of numbers is said to be $\lambda$-statistically convergent of order $\alpha$ if there is a number $L$ such that

$$
\lim _{n \rightarrow \infty} \frac{1}{\lambda_{n}^{\alpha}}\left|\left\{k \in I_{n}:\left|x_{k}-L\right| \geq \epsilon\right\}\right|=0 .
$$

In this case, we write $S_{\lambda}^{\alpha}-\lim _{k \rightarrow \infty} x_{k}=L$.

We next quote the following definition due to Mursaleen and Noman [19] on $\mu$ convergent series.

Definition 4 ([19]). Let $\mu=\left(\mu_{k}\right)$ be a sequence of positive real numbers tending to infinity such that

$$
0<\mu_{0}<\mu_{1}<\mu_{2} \ldots \text { and } \mu_{k} \rightarrow \infty \text { as } k \rightarrow \infty .
$$

Then a sequence $x=\left(x_{k}\right)$ of numbers is said to be $\mu$-convergent to a number $l$ if $\Lambda x_{k} \rightarrow l$ as $k \rightarrow \infty$, where

$$
\Lambda x_{k}=\frac{1}{\mu_{k}} \sum_{i=0}^{k}\left(\mu_{i}-\mu_{i-1}\right) x_{i}
$$

Esi and Braha [4] used Definition 4 to introduce a new notion called $\Lambda$-statistical convergence in random 2-normed spaces and studied some of its properties. Before we proceed further it would be better to recall the ideas of probabilistic and random 2-normed spaces which are of much interest in the study of random operator equations. The concept of probabilistic normed spaces was initially introduced by A. N. Sherstnev [22] in 1962. Menger [13] introduced the notion of probabilistic metric spaces in 1942. The idea of Menger [13] was to use distribution function instead of non-negative real numbers as values of the metric. In last few years these spaces 
are grown up rapidly and many detereministic results of linear normed spaces are obtained for probabilistic normed spaces. For a detailed study on probabilistic functional analysis, we refer [1, 10,11, 18,21]. In 2005, Goleț [9] used the concept of 2-norm of Gähler [8] and presented generalized probabilistic normed space which he called random 2-normed space.

Let $\mathbb{R}$ denotes the set of reals and $\mathbb{R}_{0}^{+}=[0, \infty)$. A function $f: \mathbb{R} \rightarrow \mathbb{R}_{0}^{+}$is called a distribution function if it is non-decreasing and left-continuous with $\inf _{t \in \mathbb{R}} f(t)=0$ and $\sup _{t \in \mathbb{R}} f(t)=1$.

We will denote the set of all distribution functions by $\mathfrak{D}$.

Also, a distance distribution function is a non decreasing function $F$ defined on $\mathbb{R}^{+}=[0, \infty]$ that satisfies $F(0)=0$ and $F(\infty)=1$, and is left continuous on $(0, \infty)$. Let $\mathfrak{D}^{+}$denotes the set of all distance distribution functions.

A triangular norm, briefly t-norm, is a binary operation $*$ on $[0,1]$ which is continuous, commutative, associative, non-decreasing and has 1 as neutral element, i.e., it is the continuous mapping $*:[0,1] \times[0,1] \rightarrow[0,1]$ such that for all $a, b, c \in[0,1]$ :

(1) $a * 1=a$,

(2) $a * b=b * a$,

(3) $c * d \geq a * b$ if $c \geq a$ and $d \geq b$,

(4) $(a * b) * c=a *(b * c)$.

The $*$ operations $a * b=\max \{a+b-1,0\}, a * b=a b$, and $a * b=\min \{a, b\}$ on $[0,1]$ are t-norms.

In following, we quote some needful definitions.

Definition 5 ([8]). Let $\mathrm{X}$ be a real vector space of dimension $d>1$ ( $d$ may be infinite). A real valued function $\|\cdot, \cdot\|: X^{2} \rightarrow \mathbb{R}$ satisfying the following conditions:

(1) $\left\|x_{1}, x_{2}\right\|=0$, if and only if $x_{1}, x_{2}$ are linearly dependent.

(2) $\left\|x_{1}, x_{2}\right\|=\left\|x_{2}, x_{1}\right\|$ for all $x_{1}, x_{2} \in X$,

(3) $\left\|\alpha x_{1}, x_{2}\right\|=|\alpha||| x_{1}, x_{2} \|$, for any $\alpha \in \mathbb{R}$ and

(4) $\left\|x_{1}+x_{2}, x_{3}\right\| \leq\left\|x_{1}, x_{3}\right\|+\left\|x_{2}, x_{3}\right\|$

is called a 2-norm and the pair $(X,\|\cdot, \cdot\|)$ is called a 2-normed space.

For example, if we take $X=\mathbb{R}^{2}$ with 2-norm $\left\|x_{1}, x_{2}\right\|=$ area of parallelogram spanned by the vectors $x_{1}, x_{2}$ which may be given explicitly by the formula

$$
\left\|x_{1}, x_{2}\right\|=\left|\operatorname{det}\left(x_{i j}\right)\right|=\operatorname{abs} .\left(\operatorname{det}\left(x_{i}, x_{j}\right)\right)
$$

where $x_{i}=\left(x_{i 1}, x_{i 2}\right) \in \mathbb{R}^{2}$ for each $i=1,2$. Then $(X,\|\cdot, \cdot\|)$ is a 2-normed space.

Definition 6 ([9]). Let $\mathrm{X}$ be a real linear space of dimension $d>1$ ( $d$ may be infinite), $\tau$ be a triangle function(a binary operation on $\mathfrak{D}^{+}$which is associative, commutative, nondecreasing and $\epsilon_{0}$ as a unit) and $\mathscr{F}: X \times X \rightarrow \mathfrak{D}^{+}$(for $x, y \in X$, $\mathscr{F}(x, y ; t)$ is the value of $\mathscr{F}(x, y)$ at $t \in \mathbb{R})$. Then $\mathcal{F}$ is called a probabilistic norm and $(X, \mathcal{F}, \tau)$ a probabilistic 2-normed space if the following conditions are satisfied: 
(1) $\mathcal{F}(x, y ; t)=H_{0}(t)$ if $x, y$ are linearly dependent, where $H_{0}(t)=0$ if $t \leq 0$ and $H_{0}(t)=1$ if $t>0$.

(2) $\mathcal{F}(x, y ; t) \neq H_{0}(t)$ if $x, y$ are linearly independent,

(3) $\mathcal{F}(x, y ; t)=\mathscr{F}(y, x ; t)$ for all $x, y \in X$,

(4) $\mathcal{F}(\alpha x, y ; t)=\mathscr{F}\left(x, y ; \frac{t}{|\alpha|}\right)$ for every $t>0, \alpha \neq 0$ and $x, y \in X$,

(5) $\mathcal{F}(x+y, z ; t) \geq \tau(\mathcal{F}(x, z ; t), \mathcal{F}(y, z ; t))$, where $x, y, z \in X$.

If (5) is replaced by $\mathcal{F}\left(x+y, z ; t_{1}+t_{2}\right) \geq \mathscr{F}\left(x, z ; t_{1}\right) * \mathscr{F}\left(y, z ; t_{2}\right)$ for all $x, y, z \in X$ and $t_{1}, t_{2} \in \mathbb{R}_{0}^{+}$then $(X, \mathcal{F}, *)$ is called a random 2-normed space.

Example 1. Every 2-normed space $(X,\|\cdot, \cdot\|)$ can be made a random 2-normed space by setting $\mathcal{F}(x, y ; t)=H_{0}(t-\|x, y\|)$ where

$$
H_{a}(t)= \begin{cases}0, & \text { if } t \leq a \\ 1, & \text { if } t>a\end{cases}
$$

for all $x, y \in X, t>0$ and $a * b=a b ; a, b \in[0,1]$.

Example 2. Let $(X, \| \cdot, \cdot||)$ be a 2-normed space with $\| x, z||=\left|x_{1} z_{2}-x_{2} z_{1}\right| ; x=$ $\left(x_{1}, x_{2}\right), z=\left(z_{1}, z_{2}\right)$ and $a * b=a b$ for all $a, b \in[0,1]$. For every $x, y \in X$ and $t>0$ we define $\mathcal{F}(x, y ; t)=\frac{t}{t+\| x, y||}$, then $(X, \mathcal{F}, *)$ is a random 2-normed space.

Definition 7 ([15]). Let $(X, \mathcal{F}, *)$ be a random 2-normed space. Then a sequence $x=\left(x_{k}\right)$ is said to be convergent to $x_{0} \in X$ with respect to the norm $\mathscr{F}$ if for every $\epsilon>0, t \in(0,1)$ and $\theta \neq z \in X$, there exists a positive integer $k_{0}$ such that $\mathcal{F}\left(x_{k}-\right.$ $\left.x_{0}, z ; \epsilon\right)>1-t$ whenever $k \geq k_{0}$. It is denoted by $\mathcal{F}-\lim x_{k}=x_{0}$.

Definition 8. $\mathrm{p}[15]]$ Let $(X, \mathcal{F}, *)$ be a random 2-normed space. Then a sequence $x=\left(x_{k}\right)$ is said to be statistically convergent or $S^{R 2 N}$ convergent to $x_{0} \in X$ with respect to the norm $\mathscr{F}$ if for every $\epsilon>0, t \in(0,1)$ and $\theta \neq z \in X$,

$$
\delta\left(\left\{k \in \mathbb{N}: \mathcal{F}\left(x_{k}-x_{0}, z ; \epsilon\right) \leq 1-t\right\}\right)=0 .
$$

In this case, we write $S^{R 2 N}-\lim x_{k}=x_{0}$.

Definition 9 ([4]). Let $(X, \mathcal{F}, *)$ be a random 2-normed space. Then a sequence $x=\left(x_{k}\right)$ is said to be $\Lambda$-statistically convergent with respect to the norm $\mathscr{F}$ provided that, for every $\epsilon>0, t \in(0,1)$ and $\theta \neq z \in X$,

$$
\delta_{\Lambda}\left(\left\{k \in I_{n}: \mathcal{F}\left(\Lambda x_{k}-x_{0}, z ; \epsilon\right) \leq 1-t\right\}\right)=0,
$$

i.e.

$$
\lim _{n \rightarrow \infty} \frac{1}{\lambda_{n}}\left|\left\{k \in I_{n}: \mathcal{F}\left(\Lambda x_{k}-x_{0}, z ; \epsilon\right) \leq 1-t\right\}\right|=0 .
$$

In this case, we write $S_{\Lambda}^{R 2 N}-\lim x_{k}=x_{0}$. 
Definition $10([4])$. Let $(X, \mathcal{F}, *)$ be a random 2-normed space. Then a sequence $x=\left(x_{k}\right)$ is said to be $\Lambda$-statistically cauchy with respect to the norm $\mathcal{F}$ provided that, for every $\epsilon>0, t \in(0,1)$ and $\theta \neq z \in X$, there exists a positive integer $k_{0}(\epsilon)$ such that for all $k, l \geq k_{0}$

$$
\delta_{\Lambda}\left(\left\{k \in I_{n}: \mathcal{F}\left(\Lambda x_{k}-\Lambda x_{l}, z ; \epsilon\right) \leq 1-t\right\}\right)=0 .
$$

In present paper, we study quite natural and new notion of $\Lambda$-statistical convergence of order $\alpha$ in random 2-normed spaces.

\section{MAIN RESULTS}

In this section, we begin with the following definitions of statistical and $\Lambda$-statistical convergence of order $\alpha$ in random 2-normed spaces.

Definition 11. A sequence $x=\left(x_{k}\right)$ in a random 2-normed space $(X, \mathcal{F}, *)$ is said to be statistically convergent of order $\alpha(0<\alpha \leq 1)$ to $x_{0} \in X$ provided that, for every $\epsilon>0, t \in(0,1)$ and $\theta \neq z \in X$,

$$
\lim _{n \rightarrow \infty} \frac{1}{n^{\alpha}}\left|\left\{k \in \mathbb{N}: \mathcal{F}\left(\Lambda x_{k}-x_{0}, z ; \epsilon\right) \leq 1-t\right\}\right|=0,
$$

or equivalently

$$
\lim _{n \rightarrow \infty} \frac{1}{n^{\alpha}}\left|\left\{k \in \mathbb{N}: \mathscr{F}\left(\Lambda x_{k}-x_{0}, z ; \epsilon\right)>1-t\right\}\right|=1 .
$$

In this case, we write $S^{\alpha}-\lim _{k \rightarrow \infty} x_{k}=x_{0}$.

Let $S^{\alpha}(X)$ denotes the set of all statistically convergent sequences of order $\alpha$ in a random 2-normed space $(X, \mathcal{F}, *)$.

Definition 12. Let $\lambda=\left(\lambda_{n}\right)$ be a non-decreasing sequence of positive real numbers tending to $\infty$ with $\lambda_{n+1} \leq \lambda_{n}+1, \lambda_{1}=1$. A sequence $x=\left(x_{k}\right)$ in a random 2normed space $(X, \mathcal{F}, *)$ is said to be $\Lambda$-statistically convergent of order $\alpha(0<\alpha \leq 1)$ to $x_{0} \in X$ provided that, for every $\epsilon>0, t \in(0,1)$ and $\theta \neq z \in X$,

$$
\left.\lim _{n \rightarrow \infty} \frac{1}{\lambda_{n}^{\alpha}} \mid k \in I_{n}: \mathcal{F}\left(\Lambda x_{k}-x_{0}, z ; \epsilon\right) \leq 1-t\right\} \mid=0,
$$

or equivalently

$$
\left.\lim _{n \rightarrow \infty} \frac{1}{\lambda_{n}^{\alpha}} \mid k \in I_{n}: \mathcal{F}\left(\Lambda x_{k}-x_{0}, z ; \epsilon\right)>1-t\right\} \mid=1,
$$

where $\lambda_{n}^{\alpha}$ denote the $\alpha$ th power of $\lambda_{n}$, i.e., $\left(\lambda_{n}^{\alpha}\right)=\left(\lambda_{1}^{\alpha}, \lambda_{2}^{\alpha}, \lambda_{3}^{\alpha}, \cdots \cdots\right)$. In this case, we write $S_{\Lambda}^{\alpha}-\lim _{k \rightarrow \infty} x_{k}=x_{0}$.

Let $S_{\Lambda}^{\alpha}(X)$ denotes the set of all $\Lambda$-statistically convergent sequences of order $\alpha$ in a random 2-normed space $(X, \mathcal{F}, *)$.

For the particular choice $\alpha=1$, Definition 12 coincides with the notion of $\Lambda$-statistical 
convergence of [4]; For $\lambda_{n}=n$, Definition 12 coincides with the notion of statistical convergence of order $\alpha$ in random 2-normed space; For $\lambda_{n}=n$ and $\alpha=1$, Definition 12 coincides with the notion of statistical convergence in random 2-normed space[15].

We next give an example that shows Definition 12 is well defined for $(0<\alpha \leq 1)$ but not for $\alpha>1$. In view of this, we need the following theorem with lemma.

Lemma 1. Let $\lambda=\left(\lambda_{n}\right)$ be a non-decreasing sequence as defined above and $(X, \mathcal{F}, *)$ be a random 2-normed space. Let $0<\alpha \leq 1$ and $x=\left(x_{k}\right)$ be a sequence in $X$. Then, for $\epsilon>0, t \in(0,1)$ and $\theta \neq z \in X$, the following statements are equivalent:

(1) $S_{\Lambda}^{\alpha}-\lim _{k \rightarrow \infty} x_{k}=x_{0}$,

(2) $\left.\lim _{n \rightarrow \infty} \frac{1}{\lambda_{n}^{\alpha}} \mid k \in I_{n}: \mathcal{F}\left(\Lambda x_{k}-x_{0}, z ; \epsilon\right) \leq 1-t\right\} \mid=0$,

(3) $\left.\lim _{n \rightarrow \infty} \frac{1}{\lambda_{n}^{\alpha}} \mid k \in I_{n}: \mathcal{F}\left(\Lambda x_{k}-x_{0}, z ; \epsilon\right)>1-t\right\} \mid=1$,

(4) $S_{\Lambda}^{\alpha}-\lim _{k \rightarrow \infty} \mathscr{F}\left(\Lambda x_{k}-x_{0}, z ; \epsilon\right)=1$.

Theorem 1. Let $(X, \mathcal{F}, *)$ be a random 2 -normed space and $0<\alpha \leq 1$ be given. If $S_{\Lambda}^{\alpha}-\lim _{k \rightarrow \infty} x_{k}=x_{0}$, then $x_{0}$ must be unique.

Proof. Suppose $S_{\Lambda}^{\alpha}-\lim _{k \rightarrow \infty} x_{k}=y_{0}$ where $y_{0} \neq x_{0}$. Given $\epsilon>0$ and $t>0$, choose $\eta>0$ such that $(1-\eta) *(1-\eta)>1-\epsilon$. For $\theta \neq z \in X$, define

$$
\begin{aligned}
& K_{1}(\eta, t)=\left\{k \in I_{n}: \mathcal{F}\left(\Lambda x_{k}-x_{0}, z ; \frac{t}{2}\right) \leq 1-\eta\right\} ; \\
& K_{2}(\eta, t)=\left\{k \in I_{n}: \mathcal{F}\left(\Lambda x_{k}-y_{0}, z ; \frac{t}{2}\right) \leq 1-\eta\right\} .
\end{aligned}
$$

Since $S_{\Lambda}^{\alpha}-\lim _{k \rightarrow \infty} x_{k}=x_{0}$ and $S_{\Lambda}^{\alpha}-\lim _{k \rightarrow \infty} x_{k}=y_{0}$, it follows for every $t>0$,

$$
\lim _{n \rightarrow \infty} \frac{1}{\lambda_{n}^{\alpha}}\left|K_{1}(\eta, t)\right|=0 \quad \text { and } \quad \lim _{n \rightarrow \infty} \frac{1}{\lambda_{n}^{\alpha}}\left|K_{2}(\eta, t)\right|=0
$$

Let $K(\eta, t)=K_{1}(\eta, t) \cup K_{2}(\eta, t)$, then clearly $\lim _{n \rightarrow \infty} \frac{1}{\lambda_{n}^{\alpha}}|K(\eta, t)|=0$ which immediately implies $\lim _{n \rightarrow \infty} \frac{1}{\lambda_{n}^{\alpha}}\left|K^{c}(\eta, t)\right|=1$. Let $k \in K^{c}(\eta, t)=K_{1}^{c}(\eta, t) \cap K_{2}^{c}(\eta, t)$. Now one can write,

$$
\begin{aligned}
\mathscr{F}\left(x_{0}-y_{0}, z ; \frac{t}{2}\right) \geq \mathcal{F}\left(\Lambda x_{k}-x_{0}, z ; \frac{t}{2}\right) * \mathcal{F}\left(\Lambda x_{k}-y_{0}, z ; \frac{t}{2}\right) \\
>(1-\eta) *(1-\eta)>1-\epsilon .
\end{aligned}
$$

Since $\epsilon$ is arbitrary, it follows that $\mathcal{F}\left(x_{0}-y_{0}, z ; \frac{t}{2}\right)=1$, for $t>0$ and $\theta \neq z \in X$. This shows that $x_{0}=y_{0}$.

Example 3. Let $X=\mathbb{R}^{2}$ with the 2-norm $\|x, z\|=\left\|x_{1} z_{2}-x_{2} z_{1}\right\|$ where $x=$ $\left(x_{1}, x_{2}\right), z=\left(z_{1}, z_{2}\right)$ and $a * b=a b$ for all $a, b \in[0,1]$. Let $\mathcal{F}(x, z ; t)=\frac{t}{t+\|x, z\|}$ 
where $x \in X, t \in(0,1)$ and $\theta \neq z \in X$. Then $\left(\mathbb{R}^{2}, \mathcal{F}, *\right)$ is a random 2-normed space. We define a sequence $x=\left(x_{k}\right)$ as follows:

$$
\Lambda x_{k}= \begin{cases}(1,0), & \text { if } k \text { is even, } \\ (0,0), & \text { if } k \text { is odd. }\end{cases}
$$

For $\epsilon>0, t \in(0,1)$, if we define

$$
\begin{array}{r}
K(\epsilon, t)=\left\{k \in I_{n}: \mathcal{F}\left(\Lambda x_{k}-\theta, z ; t\right) \leq 1-\epsilon\right\}, \theta=(0,0) \\
=\left\{k \in I_{n}: \frac{t}{t+\left\|\Lambda x_{k}-\theta, z\right\|} \leq 1-\epsilon\right\} \\
=\left\{k \in I_{n}:\left\|\Lambda x_{k}-\theta, z\right\| \geq \frac{\epsilon t}{1-\epsilon}>0\right\} \\
=\left\{k \in I_{n}: \Lambda x_{k}=(1,0)\right\} \\
=\left\{k \in I_{n}: k \text { is even }\right\} ;
\end{array}
$$

then,

$$
\lim _{n \rightarrow \infty} \frac{1}{\lambda_{n}^{\alpha}}|K(\epsilon, t)|=\lim _{n \rightarrow \infty} \frac{1}{\lambda_{n}^{\alpha}} \mid\left\{k \in I_{n}: k \text { is even }\right\} \mid \leq \lim _{n \rightarrow \infty} \frac{\left[\sqrt{\lambda_{n}}\right]+1}{2 \lambda_{n}^{\alpha}}=0
$$

for $\alpha>1$.

Similarly, for $\epsilon>0$ and $t \in(0,1)$ if we define

$$
B(\epsilon, t)=\left\{k \in I_{n}: \mathcal{F}\left(\Lambda x_{k}-x_{0}, z ; t\right) \leq 1-\epsilon\right\}, x_{0}=(1,0)
$$

then

$$
\lim _{n \rightarrow \infty} \frac{1}{\lambda_{n}^{\alpha}}|B(\epsilon, t)|=\lim _{n \rightarrow \infty} \frac{1}{\lambda_{n}^{\alpha}} \mid\left\{k \in I_{n}: \mathrm{k} \text { is odd }\right\} \mid \leq \lim _{n \rightarrow \infty} \frac{\left[\sqrt{\lambda_{n}}\right]+1}{2 \lambda_{n}^{\alpha}}=0
$$

for $\alpha>1$.

This shows that $S_{\Lambda}^{\alpha}-\lim _{k} x_{k}$ is not unique and we obtain a contradiction to Theorem 1.

Theorem 2. Let $(X, \mathcal{F}, *)$ be a random 2 -normed space and $0<\alpha \leq 1$ be given. For a sequence $x=\left(x_{k}\right)$ in $X$ if $\mathscr{F}_{\Lambda}-\lim _{k} x_{k}=x_{0}$, then $S_{\Lambda}^{\alpha}-\lim _{k} x_{k}=x_{0}$. However, the converse need not be true in general.

Proof. Since $\mathcal{F}_{\Lambda}-\lim _{k} x_{k}=x_{0}$, so for $\epsilon>0, t \in(0,1)$ and $\theta \neq z \in X$ there exists a positive integer $n_{0}$ such that $\mathscr{F}\left(\Lambda x_{k}-x_{0}, z ; t\right)>1-\epsilon$ for all $k \geq n_{0}$. Hence the set

$$
K(\epsilon, t)=\left\{k \in I_{n}: \mathcal{F}\left(\Lambda x_{k}-x_{0}, z ; t\right) \leq 1-\epsilon\right\} \subset\left\{1,2,3, \ldots, n_{0}-1\right\},
$$

for which we have,

$$
\lim _{n \rightarrow \infty} \frac{1}{\lambda_{n}^{\alpha}}\left|\left\{k \in I_{n}: \mathcal{F}\left(\Lambda x_{k}-x_{0}, z ; t\right) \leq 1-\epsilon\right\}\right|=0 .
$$


This shows that $S_{\Lambda}^{\alpha}-\lim _{k} x_{k}=x_{0}$. We next give the following example which shows that the converse need not be true.

Example 4. Consider the random 2-normed space as in Example 3. Define a sequence $x=\left(x_{k}\right)$ as follows:

$$
\Lambda x_{k}= \begin{cases}(k, 0), & \text { if } n-\left[\sqrt{\lambda_{n}}\right]+1 \leq k \leq n, \\ (0,0), & \text { otherwise. }\end{cases}
$$

For $\epsilon>0$ and $t>0$ if we define $K(\epsilon, t)=\left\{k \in I_{n}: \mathcal{F}\left(\Lambda x_{k}, z ; t\right) \leq 1-\epsilon\right\}$, then one can write as in Example $3 K(\epsilon, t)=\left\{k \in I_{n}: n-\left[\sqrt{\lambda_{n}}\right]+1 \leq k \leq n\right\}$

Thus,

$$
\begin{gathered}
\lim _{n \rightarrow \infty} \frac{1}{\lambda_{n}^{\alpha}}|K(\epsilon, t)|=\lim _{n \rightarrow \infty} \frac{1}{\lambda_{n}^{\alpha}}\left|\left\{k \in I_{n}: n-\left[\sqrt{\lambda_{n}}\right]+1 \leq k \leq n\right\}\right| \\
\leq \lim _{n \rightarrow \infty} \frac{\left[\sqrt{\lambda_{n}}\right]}{\lambda_{n}^{\alpha}}=0
\end{gathered}
$$

for $\frac{1}{2}<\alpha \leq 1$. This shows that $S_{\Lambda}^{\alpha}-\lim _{k} x_{k}=0$. But $\mathscr{F}_{\Lambda}-\lim _{k} x_{k} \neq 0$, since

$$
\mathscr{F}\left(\Lambda x_{k}, z ; t\right)=\frac{t}{t+\left\|\Lambda x_{k}, z\right\|}= \begin{cases}\frac{t}{t+k z_{2}}, & \text { if } n-\left[\sqrt{\lambda_{n}}\right]+1 \leq k \leq n, \\ 1, & \text { otherwise. }\end{cases}
$$

which implies

$$
\lim _{k \rightarrow \infty} \mathcal{F}\left(\Lambda x_{k}, z ; t\right)= \begin{cases}0, & \text { if } n-\left[\sqrt{\lambda_{n}}\right]+1 \leq k \leq n, \\ 1, & \text { otherwise. }\end{cases}
$$

Theorem 3. Let $(X, \mathcal{F}, *)$ be a random 2 -normed space and $0<\alpha \leq 1$ be given. Let $x=\left(x_{k}\right)$ and $y=\left(y_{k}\right)$ be two sequences in $X$.

(i) If $S_{\Lambda}^{\alpha}-\lim _{k \rightarrow \infty} x_{k}=x_{0}$ and $0 \neq c \in \mathbb{R}$, then $S_{\Lambda}^{\alpha}-\lim _{k \rightarrow \infty} c x_{k}=c x_{0}$.

(ii) If $S_{\Lambda}^{\alpha}-\lim _{k \rightarrow \infty} x_{k}=x_{0}$ and If $S_{\Lambda}^{\alpha}-\lim _{k \rightarrow \infty} y_{k}=y_{0}$, then $S_{\Lambda}^{\alpha}-\lim \left(x_{k}+\right.$ $\left.y_{k}\right)=x_{0}+y_{0}$.

Proof. The proof of the Theorem is not so hard so is omitted here.

Theorem 4. Let $(X, \mathcal{F}, *)$ be a random 2-normed space and $0<\alpha \leq \beta \leq 1$ be given. Then $S_{\Lambda}^{\alpha}(X) \subset S_{\Lambda}^{\beta}(X)$ and the inclusion is strict for some $\alpha$ and $\beta$ such that $\alpha<\beta$.

Proof. If $0<\alpha \leq \beta \leq 1$, then for every $\epsilon>0, t>0$ and $\theta \neq z \in X$, we have

$$
\begin{aligned}
& \frac{1}{\lambda_{n}^{\beta}}\left|\left\{k \in I_{n}: \mathcal{F}\left(\Lambda x_{k}-l, z ; t\right) \leq 1-\epsilon\right\}\right| \\
\leq & \frac{1}{\lambda_{n}^{\alpha}}\left|\left\{k \in I_{n}: \mathcal{F}\left(\Lambda x_{k}-l, z ; t\right) \leq 1-\epsilon\right\}\right| ;
\end{aligned}
$$


which immediately implies the inclusion $S_{\Lambda}^{\alpha}(X) \subset S_{\Lambda}^{\beta}(X)$. We next give an example that shows the inclusion in $S_{\Lambda}^{\alpha}(X) \subset S_{\Lambda}^{\beta}(X)$ is strict for some $\alpha$ and $\beta$ with $\alpha<\beta$.

Example 5. Let $\left(\mathbb{R}^{2}, \mathcal{F}, *\right)$ be a random 2-normed space as defined above. We define a sequence $x=\left(x_{k}\right)$ as follows:

$$
\Lambda x_{k}= \begin{cases}(1,0), & \text { if } n-\left[\sqrt{\lambda_{n}}\right]+1 \leq k \leq n, \\ (0,0), & \text { otherwise. }\end{cases}
$$

Then one can easily see $S_{\Lambda}^{\beta}-\lim _{k} x_{k}=0$, i.e., $x \in S_{\Lambda}^{\beta}(X)$ for $\frac{1}{2}<\beta \leq 1$ but $x \notin$ $S_{\Lambda}^{\alpha}(X)$ for $0<\alpha \leq \frac{1}{2}$. This shows that the inclusion in $S_{\Lambda}^{\alpha}(X) \subset S_{\Lambda}^{\beta}(X)$ is strict.

Theorem 5. Let $(X, \mathcal{F}, *)$ be a random 2 -normed space and $0<\alpha \leq 1$ be given. If $x=\left(x_{k}\right)$ be a sequence in $X$, then $S_{\Lambda}^{\alpha}-\lim _{k} x_{k}=x_{0}$ if and only if there exists a subset $K=\left\{k_{m}: k_{1}<k_{2}<\ldots\right\}$ of $\mathbb{N}$ such that $\lim _{n \rightarrow \infty} \frac{1}{\lambda_{n}^{\alpha}}|K|=1$ and $\widetilde{F}_{\Lambda}-$ $\lim _{k} x_{k}=x_{0}$.

Proof. First suppose that $S_{\Lambda}^{\alpha}-\lim _{k} x_{k}=x_{0}$. For $t>0, \theta \neq z \in X$ and $p \in \mathbb{N}$, if we define

$$
\begin{array}{r}
K(p, t)=\left\{k \in I_{n}: \mathcal{F}\left(\Lambda x_{k}-x_{0}, z ; t\right) \leq 1-\frac{1}{p}\right\} \\
M(p, t)=\left\{k \in I_{n}: \mathcal{F}\left(\Lambda x_{k}-x_{0}, z ; t\right)>1-\frac{1}{p}\right\} ;
\end{array}
$$

then,

$$
\lim _{n \rightarrow \infty} \frac{1}{\lambda_{n}^{\alpha}}|K(p, t)|=0
$$

Also, for $p=1,2,3, \ldots$

$$
M(1, t) \supset M(2, t) \supset \ldots M(i, t) \supset M(i+1, t) \supset \ldots
$$

and

$$
\lim _{n \rightarrow \infty} \frac{1}{\lambda_{n}^{\alpha}}|M(p, t)|=1 .
$$

Now, to prove the result in one way, it is sufficient to prove that $\mathscr{F}_{\Lambda}-\lim _{k} x_{k}=x_{0}$ over $M(p, t)$. Suppose $x_{k}$ is not convergent to $x_{0}$ over $M(p, t)$ with respect to the norm $\mathcal{F}_{\Lambda}$. Then, there exists some $\eta>0$ such that

$$
\left\{k \in \mathbb{N}: \mathcal{F}\left(\Lambda x_{k}-x_{0}, z ; t\right) \leq 1-\eta\right\}
$$

for infinitely many terms $x_{k}$. Let

$$
M(\eta, t)=\left\{k \in I_{n}: \mathcal{F}\left(\Lambda x_{k}-x_{0}, z ; t\right)>1-\eta\right\}
$$


and $\eta>\frac{1}{p}$ for $p=1,2,3 \cdots$. This implies that $\lim _{n \rightarrow \infty} \frac{1}{\lambda_{n}^{\alpha}}|M(\eta, t)|=0$. Also from (2.1), we have $K_{1}(p, t) \subset M(\eta, t)$ which gives that $\lim _{n \rightarrow \infty} \frac{1}{\lambda_{n}^{\alpha}}|M(p, t)|=0$, this contradicts (2.2). Hence $\mathscr{F}_{\Lambda}-\lim _{k} x_{k}=x_{0}$.

Conversely, suppose that there exists a subset $K=\left\{k_{m}: k_{1}<k_{2}<\ldots\right\}$ of $\mathbb{N}$ such that $\lim _{n \rightarrow \infty} \frac{1}{\lambda_{n}^{\alpha}}|K|=1$ and $\mathscr{F}_{\Lambda}-\lim _{k} x_{k}=x_{0}$. Then for every $t>0, \epsilon>0$ and $\theta \neq z \in X$ there exists a positive integer $k_{0}$ such that

$$
\left\{k \in I_{n}: \mathcal{F}\left(\Lambda x_{k}-x_{0}, z ; t\right)>1-\epsilon\right\}
$$

for all $k \geq k_{0}$. Since the set $\left\{k \in I_{n}: \mathcal{F}\left(\Lambda x_{k}-x_{0}, z ; t\right) \leq 1-\epsilon\right\}$ is contained in $\mathbb{N}-\left\{k_{0}+1, k_{0}+2, k_{0}+3, \cdots\right\}$ therefore,

$$
\lim _{n \rightarrow \infty} \frac{1}{\lambda_{n}^{\alpha}}\left|\left\{k \in I_{n}: \mathcal{F}\left(\Lambda x_{k}-x_{0}, z ; t\right) \leq 1-\epsilon\right\}\right|=0 .
$$

Hence, $S_{\Lambda}^{\alpha}-\lim _{k} x_{k}=x_{0}$.

Definition 13. Let $(X, \mathcal{F}, *)$ be a random 2-normed space. A sequence $x=\left(x_{k}\right)$ is said to be $\Lambda$-statistically Cauchy of order $\alpha(0<\alpha \leq 1)$ if for every $\epsilon>0, t \in(0,1)$ and $\theta \neq z \in X$ there exists a positive integer $k_{0}$ such that for all $k, l \geq k_{0}$

$$
\left.\lim _{n \rightarrow \infty} \frac{1}{\lambda_{n}^{\alpha}} \mid k \in I_{n}: \mathcal{F}\left(\Lambda x_{k}-\Lambda x_{l}, z ; \epsilon\right) \leq 1-t\right\} \mid=0,
$$

or equivalently

$$
\left.\lim _{n \rightarrow \infty} \frac{1}{\lambda_{n}^{\alpha}} \mid k \in I_{n}: \mathscr{F}\left(\Lambda x_{k}-\Lambda x_{l}, z ; \epsilon\right)>1-t\right\} \mid=1 .
$$

Theorem 6. Let $(X, \mathcal{F}, *)$ be a random 2 -normed space and $0<\alpha \leq 1$ be given. Then a sequence $x=\left(x_{k}\right)$ is said to be $\Lambda$-statistically convergent of order $\alpha$ iff it is $\Lambda$-statistically Cauchy of order $\alpha$.

Proof. Let $x=\left(x_{k}\right)$ be a $\Lambda$-statistically convergent sequence of order $\alpha$. Suppose that $S_{\Lambda}^{\alpha}-\lim _{k} x_{k}=x_{0}$. Let $\epsilon>0$. Choose $r>0$ such that $(1-r) *(1-r)>1-\epsilon$. If we define

$$
K(r, t)=\left\{k \in I_{n}: \mathcal{F}\left(\Lambda x_{k}-x_{0}, z ; \frac{t}{2}\right) \leq 1-r\right\},
$$

then

$$
K^{c}(r, t)=\left\{k \in I_{n}: \mathcal{F}\left(\Lambda x_{k}-x_{0}, z ; \frac{t}{2}\right)>1-r\right\} ;
$$

which gives by virtue of $S_{\Lambda}^{\alpha}-\lim _{k} x_{k}=x_{0}$,

$$
\lim _{n \rightarrow \infty} \frac{1}{\lambda_{n}^{\alpha}}|K(r, t)|=0 \quad \text { and } \quad \lim _{n \rightarrow \infty} \frac{1}{\lambda_{n}^{\alpha}}\left|K^{c}(r, t)\right|=1 .
$$


Let $m \in K^{c}(r, t)$, then $\mathcal{F}\left(\Lambda x_{m}-x_{0}, z ; \frac{t}{2}\right)>1-r$. If we take

$$
B(\epsilon, t)=\left\{k \in I_{n}: \mathcal{F}\left(\Lambda x_{k}-\Lambda x_{m}, z ; t\right) \leq 1-\epsilon\right\},
$$

then to prove the first part it is sufficient to prove that $B(\epsilon, t) \subset K(r, t)$. Let $k \in$ $B(\epsilon, t)$, which gives $\mathcal{F}\left(\Lambda x_{k}-\Lambda x_{m}, z ; t\right) \leq 1-\epsilon$. Suppose $k \notin K(r, t)$, then $\mathcal{F}\left(\Lambda x_{k}-x_{0}, z ; \frac{t}{2}\right)>1-r$. Now, we can observe that

$$
\begin{gathered}
1-\epsilon \geq \mathcal{F}\left(\Lambda x_{k}-\Lambda x_{m}, z ; t\right) \geq \mathcal{F}\left(\Lambda x_{k}-x_{0}, z ; \frac{t}{2}\right) * \mathcal{F}\left(\Lambda x_{m}-x_{0}, z ; \frac{t}{2}\right) \\
\geq(1-r) *(1-r)>1-\epsilon .
\end{gathered}
$$

This contradiction shows that $B(\epsilon, t) \subset K(r, t)$ and therefore, one way of the theorem is proved.

Conversely, suppose that $x=\left(x_{k}\right)$ is $\Lambda$-statistically Cauchy sequence of order $\alpha$ but not $\Lambda$-statistically convergent of order $\alpha$ with respect to $\mathcal{F}$. Then for every $t>0$, $\epsilon>0$ and $\theta \neq z \in X$ there exists a positive integer $m$ such that

$$
\lim _{n \rightarrow \infty} \frac{1}{\lambda_{n}^{\alpha}}|K(\epsilon, t)|=0 \text { where } K(\epsilon, t)=\left\{k \in I_{n}: \mathcal{F}\left(\Lambda x_{k}-\Lambda x_{m}, z ; t\right) \leq 1-\epsilon\right\} .
$$

This implies that $\lim _{n \rightarrow \infty} \frac{1}{\lambda_{n}^{\alpha}}\left|K^{c}(\epsilon, t)\right|=1$. Choose $r>0$ such that $(1-r) *(1-$ $r)>1-\epsilon$. Let

$$
B(r, t)=\left\{k \in I_{n}: \mathcal{F}\left(\Lambda x_{k}-x_{0}, z ; \frac{t}{2}\right)>1-r\right\} .
$$

Let $m \in B(r, t)$, then $\mathcal{F}\left(\Lambda x_{m}-x_{0}, z ; \frac{t}{2}\right)>1-r$.

Since

$$
\begin{aligned}
\mathcal{F}\left(\Lambda x_{k}-\Lambda x_{m}, z ; t\right) & \geq \mathcal{F}\left(\Lambda x_{k}-x_{0}, z ; \frac{t}{2}\right) * \mathcal{F}\left(\Lambda x_{m}-x_{0}, z ; \frac{t}{2}\right) \\
> & (1-r) *(1-r)>1-\epsilon ;
\end{aligned}
$$

therefore,

$$
\lim _{n \rightarrow \infty} \frac{1}{\lambda_{n}^{\alpha}}\left|\left\{k \in I_{n}: \mathcal{F}\left(\Lambda x_{k}-\Lambda x_{m}, z ; t\right)>1-\epsilon\right\}\right|=0 .
$$

i.e. $\lim _{n \rightarrow \infty} \frac{1}{\lambda_{n}^{\alpha}}\left|K^{c}(\epsilon, t)\right|=0$ which leads to a contradiction. Hence $x=\left(x_{k}\right)$ is $\Lambda$-statistically convergent of order $\alpha$.

\section{ACKNOWLEDGEMENT}

We would like to thank the anonymous referees of the paper for their valuable suggestions and constructive comments which definitely improve the readability of the paper. 


\section{REFERENCES}

[1] A. Aghajani and K. Nourouzi, "Convex sets in probabilistic normed spaces," Chaos, Solitons and Fractals, vol. 36, no. 2, pp. 322-328, 2008, doi: 10.1016/j.chaos.2006.06.051.

[2] R. Çolak and c. A. Bektaş, " $\lambda$-statistical convergence of order alpha," Acta Mathematica Scientia, vol. 31 B, no. 3, pp. 953-959, 2011.

[3] J. Connor, "The statistical and strong p-cesàro convergence of sequences," Analysis, vol. 8, pp. 47-63, 1988, doi: 10.1524/anly.1988.8.12.47.

[4] A. Esi and N. L. Braha, "On $\lambda$-statistical convergence in random 2-normed space," Mathematical Sciences, vol. 6, p. 62, 2012, doi: 10.1186/2251-7456-6-62.

[5] H. Fast, "Surla convergence statistique," Colloquium Mathematicum, vol. 2, pp. 241-244, 1951.

[6] J. A. Fridy, "On statistical convergence," Analysis, vol. 5, pp. 301-313, 1985, doi: 10.1524/anly.1985.5.4.301.

[7] J. A. Fridy, "Statistical limit points," Proceeding of American Mathematical Society, vol. 118, pp. 1187-1192, 1993, doi: 10.1090/S0002-9939-1993-1181163-6.

[8] S. Gähler, "2-metrische raume and ihre topologische struktur," Math. Nachr., vol. 26, pp. 115-148, 1963, doi: 10.1002/mana.19630260109.

[9] I. Goleţ, “On probabilistic 2-normed spaces,” Novi Sad J. Math., vol. 35, pp. 95-102, 2006.

[10] S. Karakus, "Statistical convergence on probabilistic normed spaces," Math. Commun., vol. 12, pp. 11-23, 2007.

[11] V. Kumar and K. Kumar, "Some inclusion relations between lacunary methods on probabilistic normed spaces," Mathematical Sciences, vol. 3, no. 3, pp. 301-312, 2009.

[12] I. J. Maddox, "Statistical convergence in a locally convex space," Math. Proc. Cambridge Philos. Soc., vol. 104, no. 1, pp. 141-145, 1988, doi: 10.1017/S0305004100065312.

[13] K. Menger, "Statistical metrics," Proc. Nat. Acad. Sci., USA, vol. 28, pp. 535-537, 1942, doi: 10.1073/pnas.28.12.535.

[14] M. Mursaleen, “ $\lambda$-statistical convergence,” Math. Slovaca, vol. 50, pp. 111-115, 2000.

[15] M. Mursaleen, "On statistical convergence in random 2-normed spaces," Acta Sci. Math.(Szeged), vol. 76, no. 1-2, pp. 101-109, 2010.

[16] M. Mursaleen, C. Çakan, S. A. Mohiuddine, and E. Savaş, "Generalised statistical convergence and statistical core of double sequences," Acta Math.Sinica Eng. Series, vol. 26, no. 11, pp. 21312144, 2010, doi: 10.1007/s10114-010-9050-2.

[17] M. Mursaleen and O. H. H. Edely, "Statistical convergence of double sequences," J. Math. Anal. Appl., vol. 288, pp. 223-231, 2003, doi: 10.1016/j.jmaa.2003.08.004.

[18] M. Mursaleen and S. A. Mohiuddine, "On ideal convergence in probabilistic normed spaces," Math. Slovaca, vol. 62, pp. 49-62, 2012, doi: 10.2478/s12175-011-0071-9.

[19] M. Mursaleen and A. K. Noman, "On the spaces of $\lambda$-convergent and bounded sequences," Thai J. Math., vol. 8, no. 2, pp. 311-329, 2010.

[20] I. J. Schoenberg, "The integrability of certain functions and related summability methods," Amer. Math. Monthly, vol. 66, pp. 361-375, 1959, doi: 10.2307/2308747.

[21] B. Schweizer and A. Sklar, "Statistical metric spaces," Pacific J. Math., vol. 10, pp. 314-344, 1960, doi: 10.2140/pjm.1960.10.313.

[22] A. N. Sherstnev, "Random normed spaces, problems of completeness," Kazan Gos. Univ. Ucen. Zap., vol. 122, pp. 3-20, 1962.

[23] H. Steinhaus, "Sur la convergence ordinaire et la convergence asymptotique," Colloq Math, vol. 2, pp. 73-74, 1951

[24] T. Šalát, "On statistically convergent sequences of real numbers," Mathematica Slovaca, vol. 30, no. 2, pp. 139-150, 1980.

[25] A. Zygmund, Trigonometric series. Cambridge, UK: Cambridge University Press, 1979. 
Authors' addresses

\section{Chawla Meenakshi}

Maharishi Markandeshwar University, Department of Mathematics, Mullana Ambala, Haryana, India

E-mail address: chawlameenakshi7@gmail.com

M. S. Saroa

Maharishi Markandeshwar University, Department of Mathematics, Mullana Ambala, Haryana, India

E-mail address: mssaroa@yahoo.com

\section{Kumar}

Haryana College of Technology and Management, Department of Mathematics, Kaithal, Haryana, India

E-mail address: vjy_kaushik@yahoo.com 